\title{
The Importance of Nutrition as the Best MEDICINE FOR EATING DisORDERS
}

\author{
| Carolyn Coker Ross, MD, MPH |
}

$\mathbf{0}$ ver seven million girls and women and one million boys and men will suffer from an eating disorder in their lifetime. Up to $3.7 \%$ of females will be diagnosed with anorexia nervosa and an estimated $4.2 \%$ will have bulimia nervosa. ${ }^{1}$ The majority of adolescent patients seen in referral centers fit into a third category, "eating disorder not otherwise specified" or EDNOS and do not fit strict criteria for either anorexia or bulimia. $^{2}$ Nineteen percent of collegeaged females are bulimic; many go undiagnosed until much later. At the other end of the spectrum, $1 \%$ to $5 \%$ of the population falls into the category of binge eating disorder, not yet an approved psychiatric diagnosis. ${ }^{3}$ Anorexics are more likely to be female (90\%-95\%); $80 \%$ of bulimics are female and $60 \%$ of BEDs are female. ${ }^{4}$ In the United States, food and weight are significant issues, as witnessed by the fact that almost half of the population is considered overweight and/or obese.

Eating disorders begin early, with $10 \%$ being diagnosed in children less than 10 years of age. One third of patients are diagnosed as preteens and adolescents up to age 15 . In total, $86 \%$ of patients are diagnosed with eating disorders before the age of 20. For this reason, the Society for Adolescent Medicine ${ }^{5}$ issued this statement: "Because of the potentially irreversible effects of an eating disorder on physical and emotional growth and development in adolescents, because of the risk of death, and because of the evidence suggesting improved outcome with early treatment, the threshold for intervention in adolescents should be lower than in adults." Irreversible risks are growth retardation, pubertal delay or arrest, impaired acquisition of peak bone mass, and increased risk of osteoporosis.

Earlier studies of eating disorders suggested disparities in the occurrence of eating disorders among different ethnic groups. Current research demonstrates that eating disorder symptoms may be as common or more common among certain ethnic groups (Asians, blacks, and Hispanics) when compared with whites. ${ }^{6}$ There was no difference found in dieting and restraint scores between Asian, Latino, and white adolescent girls and boys ${ }^{7}$ and no difference in binging or BED in obese patients who sought to lose weight with bariatric surgery. ${ }^{8}$ These changes may be related to an extension of cultural ideals in these ethnic populations of what is attractive as seen through the public media, or may represent past underreporting. However, an analysis of 18 studies (1987-2001) concluded that African-American women were less likely than white women to have an eating disorder. ${ }^{9}$ As well, a study in school age girls demonstrated that Native American girls had higher rates of restricting/purging and dieting than white or nonwhite/non-Native American populations. ${ }^{10}$

Eating disorders have one of the highest death rates of all psychiatric diagnoses. The number of deaths in anorexics is 11.6 times what would be expected in others of their same age and sex; for bulimics, this number is 1.3 times greater than expected. The most shocking number is the 56.9 times increased number of observed versus expected deaths in anorexics from suicide. If either of these groups abused alcohol, the death rates were even higher. ${ }^{11}$ Depression is a major risk factor for both substance use disorder and bulimia and explains to a great degree the association between substance use disorder and eating disorders. ${ }^{12}$ A meta-analysis by Sullivan ${ }^{13}$ found 178 deaths in 3,006 patients: 54\% died from complications of their eating disorder, 27\% from suicide, and 19\% from other and/or unknown causes. The overall rate of $.56 \%$ mortality per year in this study was 12 times greater than the expected mortality rate for women aged 15 to 24 , and the suicide rate was 75 times higher.

Medical consequences of eating disorders include arrested sexual maturity and growth failure in prepubertal patients. Many with eating disorders may look and feel deceptively well and may have normal electrograms but are still at high risk for cardiac arrhythmias and sudden death. Prolonged amenorrhea is associated with an increased risk of osteopenia and rate of fractures. Neuroimaging studies with computerized tomography (CT) have demonstrated structural brain abnormalities in both anorexics and bulimics. ${ }^{14,15}$ Bulimia is associated with electrolyte, fluid, and mineral imbalance; increased cardiac risk; gastrointestinal bleeding; dental enamel erosion; peripheral muscle weakness; cardiomyopathy; and hypometabolism. ${ }^{16}$ Despite normal weight, bulimics and BED patients may be severely malnourished.

The effects of poor nutrition are nowhere as pronounced as in the case of eating disorders. Although many may think that undernutrition or malnutrition is a side effect of eating disorders, studies have documented that it can be the cause of many of the symptoms seen in eating disorders. The earliest study to demonstrate this was done by Ancel Keys and his colleagues at the University of Minnesota in 1950. ${ }^{17}$ Young, healthy, psychologically normal males were recruited for the study as an alternative to military service. Of 100 who applied, 36 were selected for being in the best physical and psychological health. This study is known as the "starvation study," and its intent was not to study eating disorders but to develop a better understanding of how best to manage the refeeding of concentration camp survivors.

In the study, the men ate normally and continued their normal activities for the first three months while their behavior, personality, and eating patterns were stud- 
ied. Over the next six months, their diet was restricted to half of what they normally ate, resulting in a $25 \%$ loss of weight. Of note, the amount of calories prescribed in the study is the same as that being used in treating obesity. After this period of weight loss, the men were slowly refed. The results of the study were reported for only 32 of the original 36 participants, as four dropped out either during or at the end of the "semistarvation" phase. All participants experienced very dramatic changes-physical, psychological, and social.

The striking changes included an increase in preoccupation with food. During the 12-week refeeding period, binging continued, and several of the men experienced nausea and vomiting. Serious binge eating occurred in a subset of volunteers. Binges consisted of up to 10,000 calories, with volunteers reporting continued hunger even after binging to the point of becoming ill. Normalization of eating behaviors took approximately five months for the majority of the volunteers.

The volunteers also experienced emotional changes, including depression, irritability, anxiety, and apathy. Two developed psychosis. These changes persisted for some time during the refeeding period. The men became increasingly isolated during the semistarvation period and reported decreased sex drive, which took almost eight months to be restored to their previous level of functioning.

There were other changes in the volunteers as well, including decreased concentration and other cognitive changes; physical changes that included decreased need for sleep; gastrointestinal problems; dizziness; headaches; noise and light sensitivity; weakness; fluid retention; cold intolerance; and difficulties with hearing and sight. There was a $40 \%$ slowing of basal metabolic rate, low body temperature, decrease in heart rate, and respiration.

This study is important because many of the symptoms seen in eating disorders were present in the starvation study, and given that the volunteers were psychologically healthy, could not be blamed on psychological problems.

There are other studies in modern research that demonstrate the importance of nutrition and its effect on symptoms of disordered eating. For example, a study done on women with either bulimia ner- vosa or BED showed that a protein supplement given three hours before a meal reduced binge eating as compared with a carbohydrate supplement. Participants in the study also consumed less food at meals and had less hunger after receiving the protein supplementation. ${ }^{18}$ A small study by Dalvit-McPhillips ${ }^{19}$ in the 1980 s showed that a nutrient-dense diet (one without blood sugar destabilizers-white flour, refined sugar, alcohol, caffeine, flavor enhancers, and decreased salt) given to bulimic women had a dramatic effect on binge behavior. This type of diet today may be consistent with a diet without foods that have a low glycemic index. Participants were also given vitamin C, a Bcomplex vitamin, and a multivitamin. Those on the nutrient-dense diet ceased binging and were able to lose weight effortlessly and remained binge free for 2.5 years. When the control group was put on the nutrient-dense diet, they also stopped binging. This study suggests that malnutrition and blood sugar-insulin level are important factors in the binging behavior of bulimics. Therefore, the use of nutrition supplements can be a valuable addition to standard dietary therapy in the treatment of eating disorders for the following reasons:

\section{REPLACING MISSING NUTRIENTS}

Studies have shown deficiencies in a number of specific nutrients in patients with eating disorders. Eating disorder patients who restrict intake of food have been found to have deficiencies in calcium, iron, riboflavin, folic acid, ${ }^{20}$ vitamins $\mathrm{A}$ and $\mathrm{C},{ }^{21}$ and vitamin $\mathrm{B} 6^{22}$ and essential fatty acids. ${ }^{23}$ It has been postulated that anorexia may be a subclinical form of pellagra, and one study showed low niacin intake in anorexics ${ }^{24}$; niacin supplementation has been shown in several case reports to improve appetite and mental state. Other small studies have shown deficiencies in eating disorder patients for vitamins D, C, and E, as well as micronutrients such as copper and zinc. ${ }^{25}$ Nutrition supplementation for eating disorders is targeted at replacing missing nutrients and treating symptoms seen in these disorders.

The $\mathrm{B}$ vitamins are implicated in the mood disorders seen in eating disorder patients. $\mathrm{B}_{12}$ and folic acid have significant mood-enhancing benefits when used alone and in combination with antide- pressants. Both low $\mathrm{B}_{12}$ and low folic acid have been noted in patients with depression. As well, in population studies, an association has been found between depression and low levels of these two vitamins. Low levels of folate have been implicated in poor response rates to standard antidepressant therapy. ${ }^{26} \mathrm{~B}$ vitamins also convert glucose into energy in the brain cells and assists in the manufacture of neurotransmitters. $B_{12}$ is essential for nerve cell health. Studies in animals document that it can slow the rate at which rats learn.

Other micronutrients have been shown to be missing in eating disorder patients. Symptoms of zinc deficiency include decrease in smell and taste, loss of appetite, mental lethargy, generalized hair loss, diarrhea, rough and dry skin, slow wound healing, and delayed puberty.

Zinc and the mineral copper must be kept in balance. Copper, calcium, and phosphorus all increase the effectiveness of zinc. Zinc is also vital in the metabolism of essential fatty acids-levels of essential fatty acids were decreased in anorexics. Essential fatty acids are important in zinc absorption, and zinc is necessary for two stages of essential fatty acid metabolism. Zinc is a cofactor in the absorption of the $B$ vitamins, as is manganese and magnesium. Zinc is vital in the regulation of gene expression, immune function, wound healing, reproduction, growth and development, behavior and learning, blood clotting, thyroid hormone function, and insulin action. In a study of adolescent anorexics, when zinc was supplemented (50 mg elemental zinc per day), there was a decrease in depression and anxiety levels as measured on the Zung Depression Scale and the State-Trait Anxiety Inventory. ${ }^{27}$ Zinc supplementation (100 mg of zinc gluconate) has been shown to help with weight gain in anorexics, increasing their body mass index twice as fast as placebo. ${ }^{28}$ Zinc also lowers the severity and duration of diarrhea associated with malnutrition.

Omega-3 fatty acids have not been specifically tested in the treatment of eating disorders but may be useful in the treatment of many of the symptoms of eating disorders. Omega-3 fatty acids are important in brain cell communication, therefore having significant effects on brain function and mood. In the overweight or obese patient, omega-3 fatty acids have been shown to lower serum triglyceride 
levels, especially in those with high triglycerides, such as diabetics. Consumption of four grams per day of omega-3 was shown in a review of human studies to reduce serum triglyceride levels by $25 \%$ to $30 \%$, increase low-density lipoprotein by $5 \%$ to $10 \%$ and high-density lipoprotein by $1 \%$ to $3 \%$. Consumption of omega-3 fatty acids has a beneficial effect on insulin sensitivity and glucose tolerance, a useful benefit for eating disorder patients who are obese or diagnosed with metabolic syndrome (central obesity, impaired glucose tolerance, hypertension, and dyslipidemia). ${ }^{29,30}$

Omega-3 fatty acids have been shown in research to reduce the incidence of sudden death syndrome from ventricular arrhythmias by $45 \%$ to $50 \%$ and all-cause mortality by $20 \% .^{31}$ Anorexics are at higher risk of sudden death, and although there have been no studies specifically addressing the use of omega-3 fatty acids in this population, it may provide benefit. ${ }^{16}$

Many studies have shown a benefit of omega-3 fatty acid to treat depression and bipolar disorder. Some epidemiological studies demonstrated that countries in which there is high consumption of fish have a lower incidence of depression and bipolar disorder. Several studies have also shown a decrease of omega-3 fatty acid (Eicosapentaenoic acid [EPA]) in patients with depression. In one study, those participants who received supplementation with one gram of ethyl-EPA achieved a $50 \%$ reduction on the Hamilton Depression Rating Scale score, which was superior to supplementation with either two grams or four grams per day. ${ }^{32}$ Omega-3 fatty acid supplements can be given along with prescription medications for depression and bipolar disorder. In a four-week, double-blind study, patients diagnosed with recurrent unipolar depression received either ethyl-EPA or placebo. Highly significant benefits were found with the addition of the ethyl-EPA versus placebo to standard antidepressant medication. $^{33}$ The 1999 Harvard study on 30 bipolar patients with a history of at least one relapse in the prior year, of whom all but eight were on medications, used 9.6 grams of fish oil capsules, the other half received olive oil. The omega-3 fatty acid group stayed in remission significantly longer-with a decrease in depression ver- sus the placebo group-but had no decrease in their mania. ${ }^{34}$

Borderline personality disorder is also common in patients with eating disorders. In one small study, patients with moderately severe borderline personality disorder treated with one gram of ethyl-EPA demonstrated less aggression and decreased depression than those on placebo. $^{35}$

\section{OSTEOPOROSIS PREVENTION}

Anorexics are at increased risk of osteoporosis due to lowered intake, being underweight, and decreased estrogen related to amenorrhea. Calcium supplementation in pubertal girls may increase peak bone mass. ${ }^{36}$ Calcium supplementation may increase the beneficial effects of physical activity on bone. ${ }^{37}$ Deficiency of vitamin D in young people can affect their ability to reach peak bone mass. ${ }^{38}$ Special risks in eating disorder patients for osteoporosis include the following:

- anorexic girls (aged 13-23) who also suffer from depression may be at higher risk for osteoporosis than those without depression; the reason for this finding is not known ${ }^{39}$

- amenorrhea in anorexic women and young girls may indicate the onset of estrogen deficiency, which can have a negative effect on bone density and peak bone mass

- undernutrition can affect bone density through deficiency of anabolic hormones such as insulinlike growth factor $\mathrm{I}$; in addition, low weight is also a risk factor for lowered bone mass. ${ }^{40}$

Other studies have shown that nutritional factors far outweigh the impact on bone mass of endocrinologic factors such as insulinlike growth factor I. ${ }^{41}$

\section{IMPROVING AND SUPPORTING DIGESTIVE FUNCTION}

Eating disorder patients commonly have complaints of constipation, diarrhea, bloating, and other digestive symptoms. These symptoms can pose a significant barrier during the refeeding process.

Probiotics are supplements that contain beneficial bacteria or yeast that are used to replace or enhance the body's naturally occurring gut flora. Probiotics are present in some foods, one example being Lactobacillus in yogurt. Probiotics in the gastrointestinal tract are affected by the use of antibiotics, excess alcohol or drugs, stress, chronic constipation, and some diseases. Probiotics are used to manage lactose intolerance by converting lactose from foods such as milk and other dairy products to lactic acid, which is better tolerated. Lactic acid bacteria have been shown to have a cholesterol-lowering benefit, possibly by breaking down bile in the gut, thus reducing its reabsorption (entering the blood as cholesterol). Foods containing lactic acid bacteria have been shown to decrease the inflammatory response, improve absorption of minerals, and improve immune function. The most common source of probiotics in food are dairy products and foods fortified with probiotics. They are also available in capsule or tablet form. Capsules may be more effective because of their ability to elude destruction in stomach acid. Certain fermented foods contain lactic acid bacteria: kefir, yogurt, sauerkraut, and kimchi. The two most common probiotics are various species of Bifidobacterium and Lactobacillus.

Studies on probiotics that are relevant to the treatment of eating disorders have reported that (1) a probiotic mixture used to treat irritable bowel syndrome reduced abdominal pain, distension, flatulence, and borborygmi ${ }^{42}$ and (2) probiotics used in those suffering from chronic constipation reduced severity of constipation and stool consistency after two weeks. ${ }^{43}$

\section{CASE}

Maria is a 27-year-old woman hospitalized for anorexia nervosa (binge-purge type), alcohol dependence, and major depression. The patient had a history of compulsive overexercise. On admission, her lab studies showed low potassium and chloride, elevated cholesterol, HDL, and LDL. Her electrocardiogram showed bradycardia. Psychological testing results showed a diagnosis of major depressive disorder, alcohol abuse, and borderline personality disorder. The patient admitted at 74\% of her ideal body weight. She complained of fatigue, loss of interest in normal activities, irritability, insomnia, anxiety, and obsessive thoughts that her food was rotting 
in her stomach. The patient was amenorrheic for one year. Medications at the time of admission included Lexapro, $30 \mathrm{mg} /$ day for depression/anxiety; Klonopin, 1 mg twice daily for anxiety; and Ambien, $10 \mathrm{mg}$ at bedtime for sleep. The patient presented with the fear of eating specific foods; once she developed the thought of rotting food, she would obsess about it and becoming increasingly distraught until she forced herself to vomit. Other negative thoughts stemmed from her eating disorder, including thoughts about being too fat, feeling disgusted with her body, and feeling guilt or shame if she allowed herself to eat more than a very small amount of food. Bone density testing showed osteoporosis in both hip and spine.

The patient was started on the following supplement regimen:

- B-complex vitamin for mood and cognitive support

- omega-3 fatty acids containing EPA and docosahexaenoic acid for mood and cognitive support

- valerian for sleep, anxiety, and for withdrawal symptoms from benzodiazepines

- osteoporosis

- weight-bearing exercise program

- calcium, magnesium, and vitamin $\mathrm{D}_{3}$

The patient's nutrition program included:

- liquid meal replacement with a 1,200 calorie meal plan, gradually increasing the amount of food and decreasing the meal replacement

- a multivitamin with copper

- digestive support

- digestive enzymes before each meal

- probiotics

The patient was involved in group and individual therapy, targeting traumatic childhood experiences and resulting chronic post-traumatic stress disorder. She was able to be compliant with her meal plan and to gain weight, with a discharge weight that was $90 \%$ of her ideal body weight. She was tapered off her benzodiazepines and Klonopin and continued on Lexapro. She was tapered off Ambien and continued on valerian for sleep. The patient continued to have obsessive thoughts when under stress but was able to see them as false and no longer became distraught when they appeared. She was able to begin a healthy exercise program with weight training and was completely abstinent from purging or drinking while in treatment. She reported a marked decrease in bloating, abdominal pain, and constipation during her current treatment, as opposed to her previous inpatient treatment. The patient's depression scores at the time of discharge showed only a minimal level of depression compared with a severe level at the time of admission. Her anxiety had decreased significantly despite terminating her benzodiazepines, and the patient learned mind-body skill for managing anxiety.

This case demonstrates the importance of nutrition and the value of nutritional supplements in the treatment of eating disorder patients.

\section{REFERENCES}

1. American Psychiatric Association Work Group on Eating Disorders. Practice guideline for the treatment of patients with eating disorders (revision). Amer J Psych. 2000; 157(1 Suppl):1-39.

2. Bunnell DW, Shenker IR, Nussbaum MP, et al. Subclinical versus formal eating disorders: Differentiating psychological features. Int J Eat Disorder. 1990;9:357-362.

3. Spitzer RL, Yanovski S, Wadden T, et al. Binge eating disorder: its further validation in a multisite study. International Journal of Eating Disorders. 1993;13:137-153.

4. National Eating Disorders Association. Available at: http://www.edap.org/p.asp? WebPage_ID=337. Accessed March 2, 2007.

5. Eating Disorders in Adolescents: Position Paper of the Society for Adolescent Medicine. J of Adolescent Health. 2003;33: 496-503.

6. Shaw H, Ramirez L, Trost A, et al. Body image and eating disturbances across ethnic groups: More similarities than differences. Psych of Addic Behav. 2004;18:12-18.

7. Cachelin FM, Weiss JW, Garbanati JA. Dieting and its relationship to smoking, acculturation, and family environment in Asian and Hispanic adolescents. Eating Disorders. 2004;11:51-61.

8. Sanchez-Johnson L, Dymek M, Alverdy J, LeGrange D. Binge eating and eating-related cognitions and behavior in ethnically diverse obese women. Obesity Research. 2003;11:1002-1009.
9. O’Neill SK. African American women and eating disturbances: A meta-analysis. Journal of Black Psychology. 2003;29:3-16.

10. Lynch WC, Eppers KD, Sherrod JR. Eating attitudes of Native American and white female adolescents: a comparison of BMIand age-matched groups. Ethnicity \& Health. 2004;9:253-266.

11. Keel PK, Dorer DJ, Eddy KT, et al. Predictors of mortality in eating disorders. Arch of Gen Psychiatry. 2003;60:179-183.

12. Stice E, Burton EM, Shaw H. Prospective relations between bulimic pathology, depression, and substance abuse: unpacking comorbidity in adolescent girls. J Consult Clin Psychol. 2004;72:62-71.

13. Sullivan PF. Mortality in anorexia nervosa. Am J Psychiatry. 1995;152:1073-1074.

14. Krieg JC, Pirke KM, Lauer C, Backmund H. Endocrine, metabolic, and cranial computed tomographic findings in AN. Biol Psychiatry. 1988;23:377-87.

15. Krieg JC, Lauer C, Pirke KM. Structural brain abnormalities in patients with bulimia nervosa. Psychiatry Research. 1989;27: 39-48.

16. NEDA. Information for treatment professionals. Available at: http://edap.org/p. asp?WebPage_ID=293. Accessed March 5, 2007.

17. Taylor HL, Keys A. Adaptation to caloric restriction. Science. 1950 Aug 25;112:215218.

18. Latner JD, Wilson GT. Binge eating and satiety in bulimia nervosa and binge eating disorder: effects of macronutrient intake. Int J Eat Disord. 2004;36:402-415.

19. Dalvit-McPhillips S. A dietary approach to bulimia. Physiol Beav. 1984;33:769-775.

20. Abou-Saleh MT, Coppen A. The biology of folate in depression: implications for nutritional hypotheses of the psychoses. J Psychiatr Res. 1986;20:91-101.

21. Beaumont PJ, Chambers TL, Rouse L, Abraham SF. The diet composition and nutritional knowledge of patients with anorexia nervosa. J Hum Nutr. 1981;35: 265-273.

22. Rock CL, Vasantharajan S. Vitamin status of eating disorder patients: relationship to clinical indices and effect of treatment. Int J Eat Disorder. 1995;18:257-262.

23. Langan SM, Farrell PM. Vitamin E, vitamin A and essential fatty acid status of patients hospitalized for anorexia nervosa. Am J Clin Nutr. 1985;41:1054-1060.

24. Thibault L, Roberge AG. The nutritional status of subjects with anorexia nervosa. Int J Vitam Nutr Res. 1987;57:447-452.

25. Humphries L, Vivian B, Stuart M, McClain CJ. Zinc deficiency and eating disorders. J Clin Psychiatry. 1989;50:456-459.

26. Coppen A, Bolander-Gouaille C. Treatment of depression: time to consider folic 
acid and vitamin B12. J Psychopharmacol. 2005;19:59-65.

27. Katz RL, Keen CL, Litt IF, et al. Zinc deficiency in anorexia nervosa. J Adolesc Health Care. 1987;8:400-406.

28. Birmingham CL, Goldner EM, Bakan R. Controlled trial of zinc supplementation in anorexia nervosa. Int J Eat Disord. 1994;15: 251-255.

29. Harris WS. N-3 fatty acids and serum lipoproteins: human studies. Am J Clin Nutr. 1997;65:1645S-1654S.

30. Ebbesson SO, Risica PM, Ebesson LO, et al. Omega-3 fatty acids improve glucose tolerance and components of the metabolic syndrome in Alaskan Eskimos: the Alaska Siberia project. Int J Circumpolar Health. 2005;64:396-408.

31. Covington MB. Omega-3 Fatty Acids. American Family Pbysician. 2004;70:133140.

32. Peet M, Horrobin DF. A dose-ranging study of the effects of ethyl-eicosapentanoate in patients with ongoing depression despite apparently adequate treatment with standard drugs. Arch Gen Psychiatry. 2002; 59:913-919.

33. Nemets B, Stahl Z, Belmaker RH. Addition of omega-3 fatty acid to maintenance med- ication treatment for recurrent unipolar depressive disorder. Am J Psychiatry. 2002; 159:477-479.

34. Stoll AL, Severus WE, Freeman MP, et al. Omega 3 fatty acids in bipolar disorder: a preliminary double-blind, placebo-controlled trial. Arch Gen Psychiatry. 1999;56: 407-412.

35. Zanarini MC, Frankenburt FR. Omega-3 Fatty Acid treatment of women with borderline personality disorder: a doubleblind, placebo-controlled pilot study. Am J Psychiatry. 2003;160:167-169.

36. Dodiuk-Gad RP, Rozen GS, Rennert G, et al. Sustained effect of short-term calcium supplementation on bone mass in adolescent girls with low calcium intake. $A m J$ Clin Nutr. 2005;81:175-188.

37. Specker BL. Evidence for an interaction between calcium intake and physical activity on changes in bone mineral density. J Bone Miner Res. 1996;11:1539-1544.

38. Calvo MS, Whiting SJ, Barton CN. Vitamin D intake: a global perspective of current status. J Nutr. 2005;135:310-316.

39. Konstantynowicz J, Kadziela-Olech H, Kaczmarski M, et al. J Clin Endocrinol MEtab. 2005;90:5382-5. Epub 2005 Jun 7.
40. Miller KK. Mechanisms by which nutritional disorders cause reduced bone mass in adults. J Womens Health (Larchmt). 2003; 12:145-150.

41. Heer M, Mika C, Grzella I, et al. Changes in bone turnover in patients with anorexia nervosa during eleven weeks of inpatient dietary treatment. Clin Chem. 2002;48: 754-760.

42. Kajander K, Hatakka K, Poussa T. A probiotic mixture alleviates symptoms in irritable bowel syndrome patients: a controlled 6-month intervention. Aliment Pharmacol Ther. 2005;22:387-394.

43. Koebnick C, Wagner I, Leitzmann P, et al. Probiotic beverage containing Lactobacillus casei Shirota improves gastrointestinal symptoms in patients with chronic constipation. Can J Gastroenterol. 2003;Nov17: 655-659.

Carolyn Coker Ross, MD, MPH, is a graduate of the residential fellowship at the University of Arizona Program in Integrative Medicine and is the chief of the Eating Disorders Program and head of the Integrative Medicine Department at Sierra Tucson in Tucson, Arizona. 